\title{
OBSERVATIONS ON THE FACILITY OF AQUEOUS OUTFLOW IN CLOSED-ANGLE GLAUCOMA*†
}

\author{
BY
}

\author{
WALLACE S. FOULDS \\ Addenbrooke's Hospital, Cambridge
}

IN closed-angle glaucoma the darkroom test may be followed by a rise in ocular tension and a measurable fall in the facility of aqueous outflow.

In an earlier communication (Foulds, 1956), it was suggested that this decrease in the facility of outflow was a more reliable guide to the occurrence of angle closure during the test, than the rise in ocular tension, particularly in early cases of closed-angle glaucoma, where a positive darkroom test was but rarely encountered. The value of similar outflow measurements in conjunction with the mydriatic test has recently been stressed by Becker and Thompson (1958). Further work on the effects of the darkroom test on the facility of outflow has confirmed the earlier opinion, and has also provided useful information about the state of the angle between hypertensive attacks. The results of this work are presented in this paper.

For the purpose of the present study, a series of 42 normal eyes and a comparable $\ddagger$ group of 97 eyes affected by closed-angle glaucoma were submitted to the "darkroom outflow test".

\footnotetext{
* Received for publication December 18, 1958.

$\dagger$ Incorporating work carried out at the Glaucoma Clinic of the Institute of Ophthalmology, London; Director, Sir Stewart Duke-Elder.

$\ddagger$ The 42 normal eyes used in this investigation were all carefully examined to exclude the presence of closed-angle or any other type of glaucoma. Each had a medium or wide angle, and normal media, fundus, and visual field. In none was there at any time any symptom suggestive of glaucoma. In the eyes affected by closed-angle glaucoma, the diagnosis was based on the presence of a narrow anterior chamber angle, together with a known hypertensive episode in either that eye or its fellow. The angles of these eyes were carefully examined, after the instillation of a miotic, if necessary, to exclude the presence of goniosynechiae. In order that the variance might not be minimized, only one eye, chosen at random, from each case, was used in the subsequent analysis. The two series were comparable as regards age and sex. In the glaucomatous series forty were male and fifty-seven female, the sex distribution in the normal series being fourteen male and twenty-eight female. A $\chi^{2}$ test failed to reveal any significant difference between the two series in this respect $\left(\chi^{2}=0 \cdot 77 ; n=1 ; P=>0 \cdot 10\right)$. Similarly, no significant differences were found in the age distributions, within or between the two series of cases. In the normal series, the mean age of the males $(56.2$ years $\pm 16 \cdot 7)$ was not significantly different from that of the females $(60.9$ years \pm 11.7$)(t=1.05 ; n=40 ; P=>0 \cdot 10)$. In the glaucomatous series the mean age of the males was 55.9 years \pm 9.2 and that of the females 56.2 years \pm 15.7 . Again there was no significant difference between these figures $(t=0 \cdot 08 ; n=95 ; P=>0 \cdot 10)$. Comparing the total mean age of all the normal cases $(59$ years $\pm 13 \cdot 5)$ with that in the glaucomatous series (56.1 years \pm 10.0$)$, it could be seen that both series were entirely comparable in age distribution $\left(t=1 \cdot 56 ; n=137 ; P=>0 \cdot 10 ; F=1 \cdot 35 ; n_{1}=41 ; n_{2}=96 ; P=>0 \cdot 10\right)$.
} 


\section{Method}

The eye to be tested was anaesthetized by the instillation of two to three drops of 1 per cent. amethocaine hydrochloride. A drop of sterile liquid paraffin was instilled to protect the cornea. The ocular tension was measured with a standard Schiötz X-tonometer, and the facility of aqueous outflow was determined by tonography (Grant, 1951). The patient was then allowed to sit for half an hour in normal room lighting, and at the end of this period the ocular tension was again measured with the same tonometer. The patient was next seated in a dark room for one hour, being cautioned not to fall asleep. At the end of this hour the ocular tension was again measured and the facility of aqueous outflow determined. The initial measurements of ocular tension and aqueous outflow were made under similar conditions of artificial lighting and the final measurements were made in standard conditions of dim illumination. All measurements were made with the patient recumbent.

\section{Results}

Normal Eyes.-There was no significant change in the facility of outflow with the test, the mean change in outflow being $\log 0.005$, which is not significantly different from zero.*

Glaucomatous Eyes.-The test resulted in a marked lowering of the facility of outflow, the mean reduction being approximately 50 per cent. of the initial figure $(\log 0 \cdot 271 \pm 0 \cdot 253)$. This large change in the facility of outflow is significantly greater than that in the series of normal eyes. $\dagger$

It was concluded that a positive result with the darkroom outflow test could be taken as a fall in the facility of outflow of more than $\log 0 \cdot 160$ (i.e. approximately 30 per cent. of the initial outflow level), this figure being three times the standard deviation of the mean change in outflow found in normal eyes with the test. Of the 97 glaucomatous eyes tested, 67 per cent. gave a positive change in outflow with the darkroom test, while only 30 per cent. gave a rise in ocular tension of more than $8 \mathrm{~mm}$. $\mathrm{Hg}$ during the same test. This difference is significant. $\$$

It may seem strange that a decrease in the facility of outflow should not always be accompanied by a significant rise in ocular tension, but it would appear that some little time is required for a rise in ocular tension to develop after closure of the angle of the anterior chamber. An attempt to verify this supposition was made by submitting some glaucomatous eyes to darkroom outflow tests of $15,30,40$, and 60 minutes' duration. Not many eyes were investigated, however, and statistical evaluation of the results was not possible. The results of this test are shown in Fig. 1 (opposite). In the eyes tested, a fall in the mean facility of outflow preceded the rise in ocular tension.

\footnotetext{
* The change in outflow was expressed as a fraction of the initial outflow level and for convenience the analysis was carried out on the difference between the logarithms of the first and second outfiow measurements. in outflow in the normal series was $\log 0.005 \pm 0.055$, not significantly different from zero. $(t=0.14 ; n=41 ; P=>0.10)$. in outflow in the normal series was L

$\dagger(t=6 \cdot 69 ; n=137 ; P=<0 \cdot 001)$.
$\ddagger\left(x^{2}=24 \cdot 6 ; n=1 ; P=<0.001\right)$.
} 


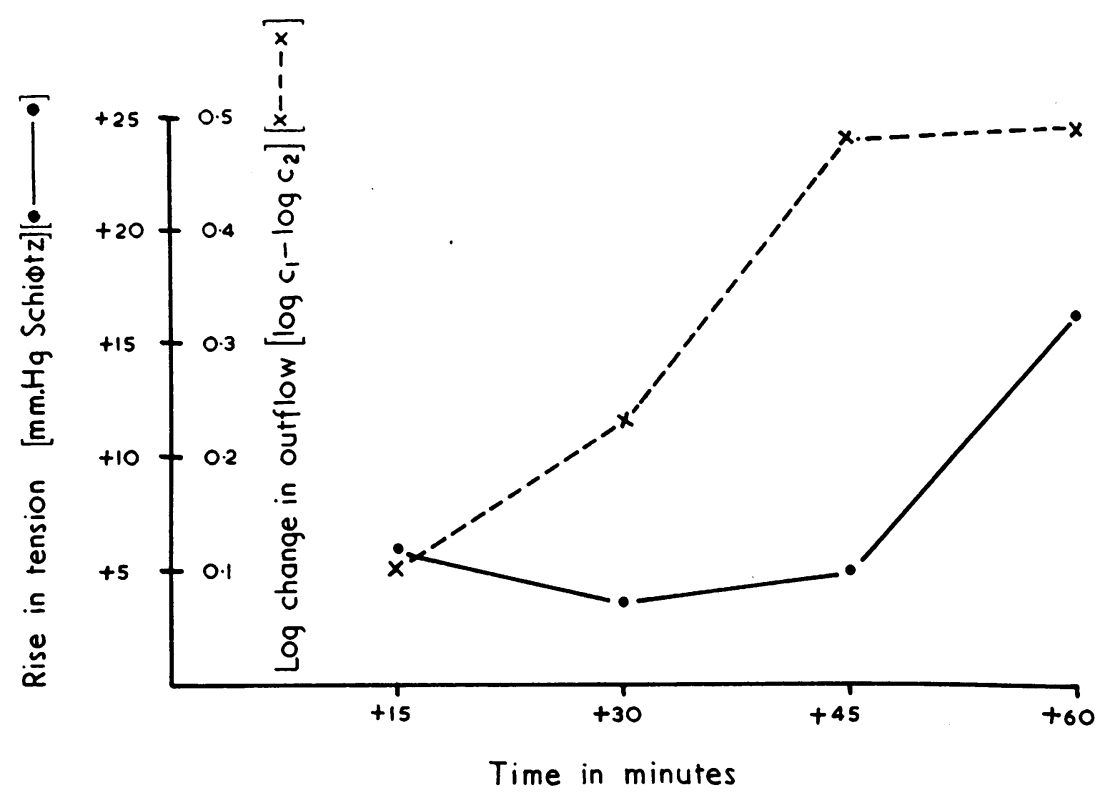

FIG. 1.-Relative mean rates of change in ocular tension and aqueous outflow in eyes with closedangle glaucoma subjected to the darkroom outflow test. There is a fall in the facility of aqueous outflow before the ocular tension rises.

It is currently accepted that in closed-angle glaucoma there is an abnormally narrow angle which, on suitable provocation, closes, so causing an acute attack of glaucoma, but which remains open and functions normally between hypertensive attacks.

It is also thought that chronic closed-angle glaucoma results from occlusion of the angle by goniosynechiae which have formed during earlier sub-acute hypertensive attacks leading to a permanent rise in the base pressure of the affected eye, cupping of the optic disc, and visual field loss.

It has, however, been suggested (Phillips, 1956; Shaffer, 1956), that in this disease the angle of the anterior chamber may be progressively closed by contact between the root of the iris and the cornea, starting in the upper narrowest sector, and that an acute attack of glaucoma may eventually result from sudden closure of the small remaining open sector of the angle. It has also been shown (Foulds and Phillips, 1957), in some cases of chronic closedangle glaucoma with permanently raised ocular tension and deeply cupped optic discs, that the angle is not necessarily occluded by goniosynechiae, but may be obstructed by reversible irido-corneal contact.

Whether it is the exception rather than the rule that, in cases of closed-angle glaucoma, the angle is partly closed by an increasing degree of irido-corneal contact long before the disease becomes otherwise manifest is difficult to determine. Gonioscopy is less helpful than might be expected, as even 
moderate degrees of contact may easily be missed because of the "on-off" phenomenon (Smith, 1954), and because of the difficulty in determining whether a very narrow and apparently closed angle is, in fact, obstructed.

The behaviour of eyes submitted to the darkroom test supports the view that partial obstruction of the angle may exist even in the presence of an apparently normal occular tension, for it has been shown (Foulds, 1957) that eyes with a "high normal" resting ocular tension are more likely to show a positive rise on provocation than eyes in which the resting tension is lower.

The results of the present investigation were examined for evidence of obstruction of the angle prior to the darkroom outflow test, and to decide whether such obstruction, if present, influenced the result of the test.

When the resting ocular tension in the group of normal eyes was plotted against the facility of aqueous outflow, no correlation could be demonstrated between these two factors. ${ }^{*}$ This suggests that in the normal eye, as might be expected, the intra-ocular pressure is not governed solely by the level of aqueous outflow, but is probably determined by the random interaction of all the factors (inflow, outflow, level of capillary blood-pressure, scleral rigidity, etc.) which may influence it (Fig. 2).

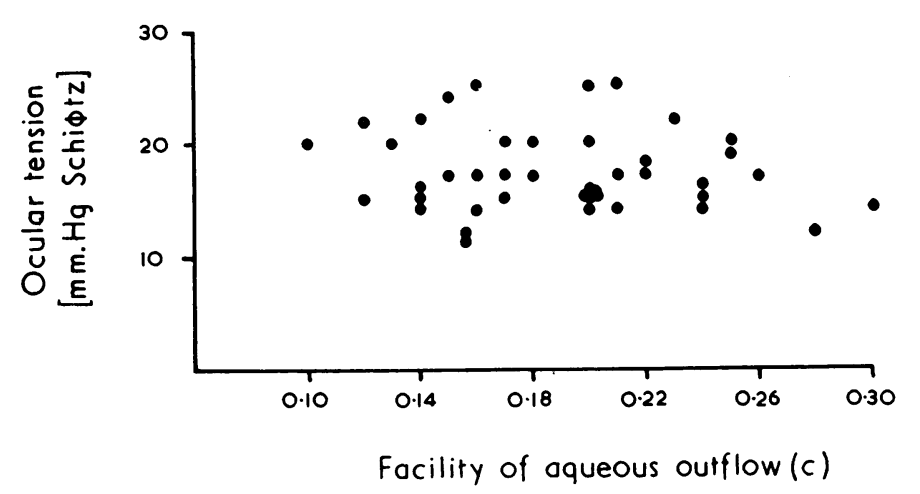

FIG. 2.- Lack of correlation between ocular tension and facility of aqueous outflow in $\mathbf{4 2}$ normal eyes. $(r=-0 \cdot 178 ; n=40 ; P=>0 \cdot 10)$.

In eyes suspected of having closed-angle glaucoma, however, a definite correlation was found between the prevailing level of aqueous outflow and that of the ocular tension $\dagger$ (Figs 3 and 4, opposite). In none of these glaucomatous eyes was the ocular tension at the time of measurement higher than $31 \mathrm{~mm}$. $\mathrm{Hg}$ (Schiötz), so that all may be regarded as having tensions within the normal range. As the glaucomatous eyes differed from those in

$$
\text { * }(r=-0.178 ; n=40 ; P=>0.10) . \quad \dagger(r=-0.455 ; n=95 ; P=<0.001) .
$$


the normal series only in being affected by the disease, it is reasonable to attribute to the disease this relationship between ocular tension and outflow.

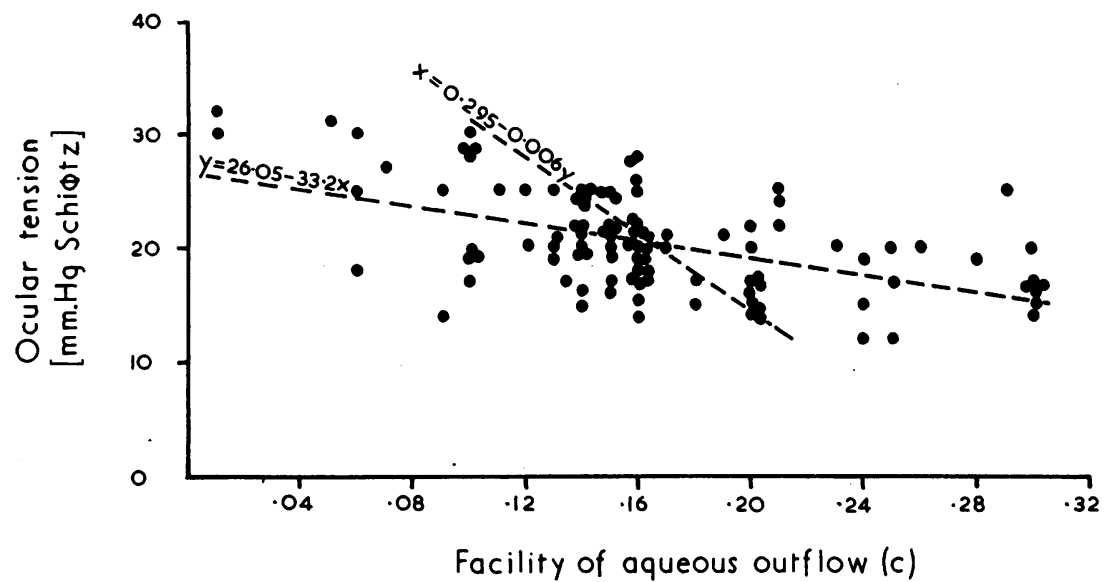

Fig. 3.-Correlation between ocular tension and facility of aqueous outflow in eyes with closedangle glaucoma. Although the ocular tension is within normal limits in each case, the higher levels of ocular tension are associated with abnormally low values of aqueous outflow. The regression lines are superimposed. $(r=-0.455 ; n=95 ; P=<0.001)$.

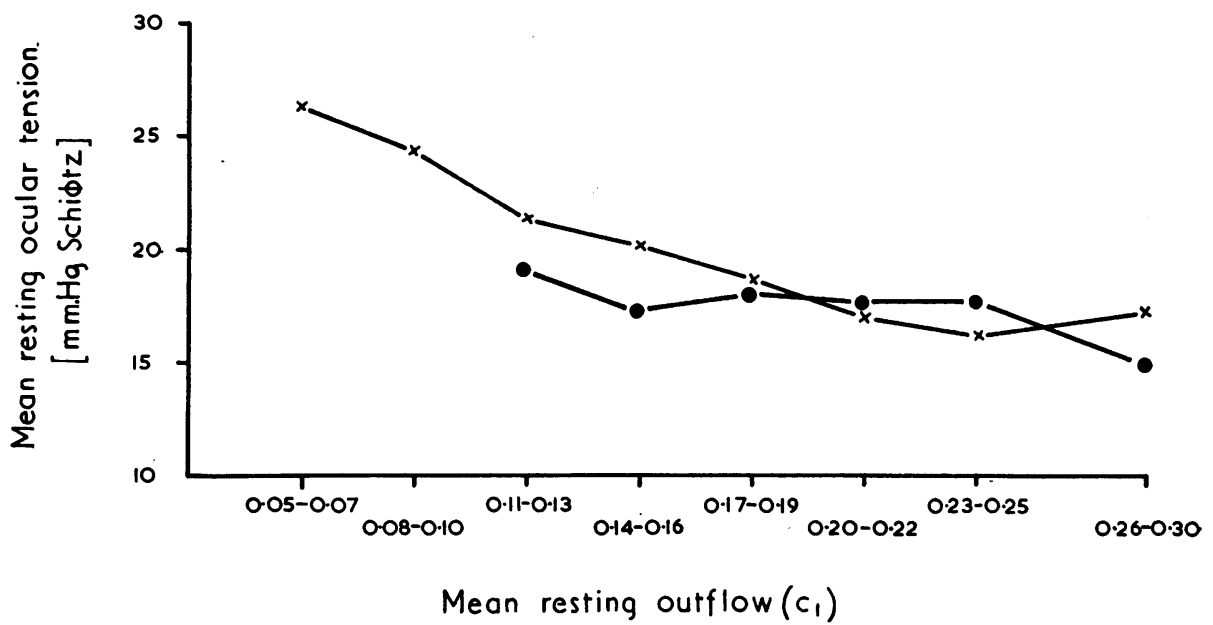

Fig. 4.-A comparison of the mean ocular tension at different levels of aqueous outflow in 42 normal eyes $(\bullet)$ and 97 normotensive eyes affected by closed-angle glaucoma $(x)$. In the latter group, lower values of outflow are present and a definite relationship exists between ocular tension and facility of outflow. This relationship is not found in the group of normal eyes.

As it is known (Grant, 1951) that closure of the angle decreases the facility of aqueous outflow, it would seem likely that the increasingly low values of outflow factor found in affected eyes in which the ocular tension was nearing the upper limits of normality could also be explained by the existence in them of some degree of angle closure. This explanation is the more likely 
when it is noted that low values of outflow factor, which were not found in any of the normal eyes, were not infrequently seen in glaucomatous eyes wherein the resting ocular tension was between 25 and $31 \mathrm{~mm}$. $\mathrm{Hg}$. In addition, a comparison of the mean level of aqueous outflow in the group of normal eyes $(c=0 \cdot 19 \pm 0 \cdot 046)$ with that in the whole group of glaucomatous eyes $(c=0 \cdot 168 \pm 0 \cdot 058)$ shows that a significantly lower mean level of aqueous outflow was present in the group affected by the disease.* As the presence of goniosynechiae in these eyes had been ruled out, any closure of the angle which was present must have been due to contact and not to adhesion between the root of the iris and the cornea.

If irido-corneal contact is present in some affected eyes, even where the ocular tension is apparently normal, one would expect such eyes to respond more readily to the provocative tests usually employed than would those in which such contact was minimal or absent. The results of the darkroom test, for instance, should be related to the pre-test outflow level.

In the present series of glaucomatous eyes submitted to the darkroom outflow test, a rise in ocular tension of more than $8 \mathrm{~mm}$. $\mathrm{Hg}$ was found in $83 \cdot 3$ per cent. of those wherein the initial level of outflow was less than $0 \cdot 12$; a similar rise occurred in only $17 \cdot 7$ per cent. of those with a higher level of outflow (Table).

TABLE

RESULTS OF DARKROOM TEST RELATED TO THE RESTING LEVEL OF AQUEOUS OUTFLOW IN EYES WITH CLOSED-ANGLE GLAUCOMA

\begin{tabular}{|c|c|c|c|c|c|c|}
\hline \multirow{3}{*}{\multicolumn{2}{|c|}{ Facility of Aqueous Outflow }} & \multirow{3}{*}{ No. of Eyes } & \multicolumn{4}{|c|}{ Result of Darkroom Test } \\
\hline & & & \multicolumn{2}{|c|}{ Positive } & \multicolumn{2}{|c|}{ Negative } \\
\hline & & & No. & Per cent. & No. & Per cent. \\
\hline Less than $0 \cdot 12 \ldots$ & . & 18 & 15 & $83 \cdot 3$ & 3 & $16 \cdot 7$ \\
\hline Greater than 0.13 & $\ldots$ & 79 & 14 & $17 \cdot 7$ & 65 & $82 \cdot 3$ \\
\hline
\end{tabular}

The darkroom test is more often positive when the facility of outflow at the start of the test is $c=0 \cdot 12$ or less than when $c=0.13$ or more. $\left(\chi^{2}=25 \cdot 85 ; n=1 ; P=<0.001\right)$.

This difference is significant. $\dagger$ The increasing incidence of positive results with the darkroom test with decreasing initial outflow levels is shown in Fig. 5 (opposite), where the percentage incidence of positive results with the test is classified according to the initially determined aqueous outflow level.

To discover whether there was a definite relationship between the magnitude of the rise in ocular tension with the darkroom test and the facility of outflow

$$
*(t=2.06 ; n=137 ; P=<0.05) . \quad \dagger\left(x^{2}=27.06 ; n=1 ; P=<0.001\right) .
$$




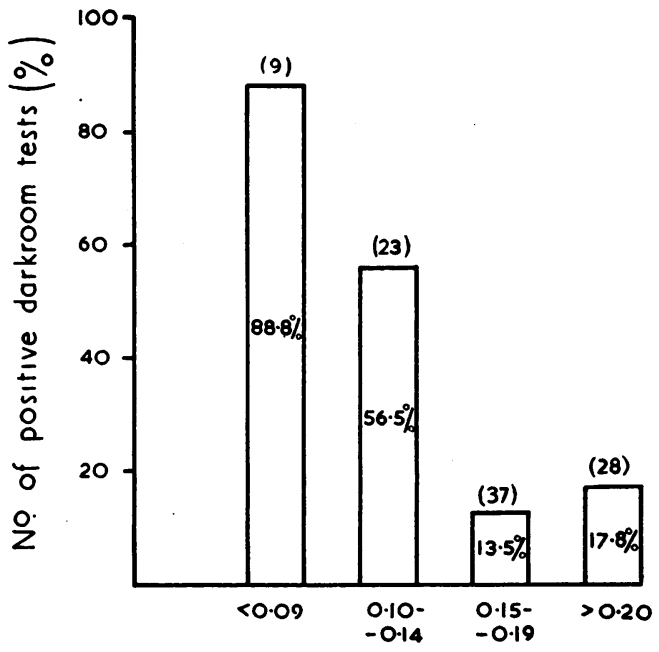

Coefficient of aqueous outflow (c)

Fig. 5.-Results of darkroom test in relation to resting facility of aqueous outflow in closed-angle glaucoma. The greatest incidence of positive results is seen when the initial level of outflow is below $c=0.09$. The initial ocular tension in each case was below $31 \mathrm{~mm}$. Hg. Figures in brackets represent numbers of cases. at the start of the test, these two factors were determined for each of the 97 glaucomatous eyes tested and plotted against each other (Fig. 6). It was apparent that the lower the initial outflow level, the higher was the rise in ocular tension, there being a significant degree of correlation between the two factors.*

A similar relationship was found to exist between the change in outflow during the period in the dark and the initial level of aqueous outflow. $\dagger$ This suggests that when the angle is largely obstructed prior to the test the remaining open sector of the angle is more sensitive to changes in illumination than is the case when all the angle is open.

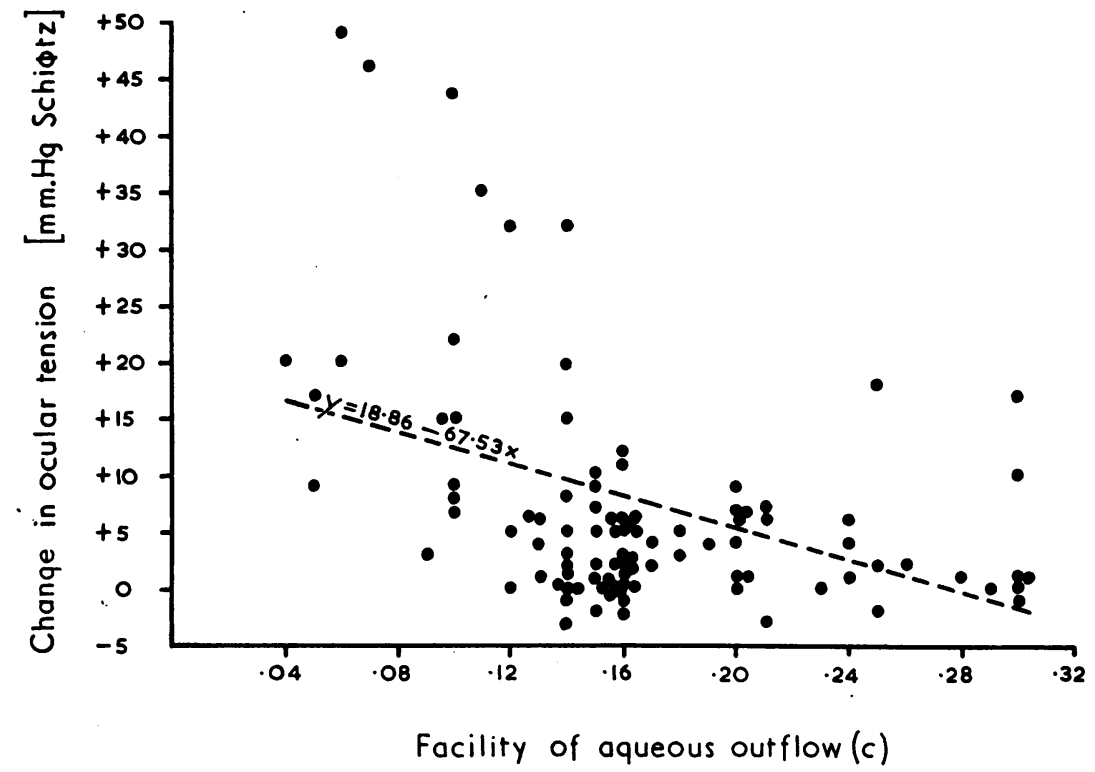

FIG. 6.-Relationship between resting facility of aqueous outflow (c) and rise in ocular tension with the darkroom test in eyes with closed-angle glaucoma. Low initial levels of outflow are associated with greater rises in ocular tension than higher levels of outflow. The regression line is indicated.

$*(r=-0.322 ; n=95 ; P=<0.01)$. $\dagger(r=-0.363 ; n=95 ; P=<0.001)$. 


\section{Discussion}

The evidence presented in this paper supports the view that the angle closure in closed-angle glaucoma is not usually an "all-or-none" phenomenon but is a gradual process which eventually involves the major part of the angle, the rate of progress depending upon slow changes in the anatomical factors determining the width of the angle together with such secondary angle-crowding factors as may affect the eye from time to time. The rate of angle closure is greater on provocation (e.g. with the darkroom test) than in normal circumstances, but the production of an appreciable degree of angle closure, measured as either a rise in ocular tension or as a fall in the facility of outflow, still requires a considerable interval.

During a provocative test, such a degree of angle closure would presumably be reached more quickly in eyes in which the angle was already partly closed, than in those in which it was unobstructed at the start of the test. This supposition is supported by the finding that eyes in which the initial facility of outflow is low more often give a positive result with the darkroom test and a greater rise in ocular tension (or a greater fall in the facility of outflow with the test) than those in which the initial outflow level is high.

That a measurable degree of obstruction to aqueous outflow (and thus of angle closure) can be demonstrated in some eyes affected by closed-angle glaucoma, even where the ocular tension is still within normal limits, is of importance in the treatment of the disease. Thus an ocular tension within the normal range in an affected eye does not necessarily indicate that this tension is normal for the eye in question, or that the angle is fully open; for the progressive occlusion of the angle which occurs in this disease is accompanied by a slow rise in base pressure, which does not exceed the upper limits of normality until a considerable area of the angle is obstructed. Gonioscopic examination of affected eyes suggests that an ocular tension of 25 to $30 \mathrm{~mm}$. $\mathrm{Hg}$ (Schiötz) usually means that about one-quarter to one-third of the angle is closed. It follows that an ocular tension within the normal range should not be regarded as proof of the efficacy of miotic therapy unless it can also be shown that the angle is at the same time fully open in its whole circumference. If doubt exists on this point, a peripheral iridectomy is indicated, as the existence of even partial angle closure is likely to predispose to the formation of goniosynechiae converting a condition readily amenable to surgical cure to one of organic obstruction of the angle which is by no means so easy to treat.

\section{Summary}

The changes in the ocular tension and facility of aqueous outflow which resulted from the darkroom test in 97 eyes with closed-angle glaucoma and 42 normal eyes have been investigated. A positive decrease in the facility of outflow with the test (at least 30 per cent. of the initial outflow level) 
occurred in 67 per cent., while a significant rise in ocular tension (more than $8 \mathrm{~mm}$. Hg) occurred in only 30 per cent. of the glaucomatous eyes.

A definite correlation was found between the resting level of ocular tension and the facility of aqueous outflow in the glaucomatous eyes, but this relationship was not present in the normal eyes. The incidence of positive results with the darkroom and darkroom outflow tests was directly related to the facility of aqueous outflow at the start of the test. These facts may be explained by the presence in affected eyes of some degree of angle closure even when the ocular tension is apparently within normal limits.

The significance of these findings is discussed in relation to the treatment of closed-angle glaucoma.

I wish to record my appreciation of the helpful criticism given by Sir Stewart Duke-Elder, and to thank Mr. T. Tarrant, of the Department of Medical Illustration of the Institute of Ophthalmology, for preparing the line drawings.

\section{REFERENCES}

BeCKer, B., and ThOMPson, H. E. (1958). Amer. J. Ophthal., 46, 305.

FouLDS, W.S. (1956). Trans. ophthal. Soc. U.K., 76, 83. (1957). Brit. J. Ophthal., 41, 200. and PHILLIPS, C. I. (1957). Ibid., 41, 208.

Grant, W. M. (1951). Trans. Amer. Acad. Ophthal. Otolaryng., 55, 774.

Phillips, C. (1956). Brit. J. Ophthal. 40, 129.

ShAFFER, R. N. (1956). In "Glaucoma: Trans. First Conf., 1955", ed. F. W. Newell, pp. 75, 63. Josiah Macy Jr. Foundation, New York.

SMITH, REDMOND (1954). Brit.J. Ophthal., 38, 136. 